\section{A) Check for updates}

Cite this: Nanoscale, 2020, 12, 13980

\title{
Inducing rapid polysulfide transformation through enhanced interfacial electronic interaction for lithium-sulfur batteries $\uparrow$
}

\author{
Chao Shen, $\star^{\mathrm{a}}$ Kun Zhang, (D) * ${ }^{\mathrm{a}}$ You You, ${ }^{\mathrm{a}}$ Hui Wang, ${ }^{\mathrm{a}}$ Ruiqi Ning, ${ }^{\mathrm{a}}$ Yaqin Qi, \\ Nan Li, ${ }^{a}$ Cuimin Ding, ${ }^{a}$ Keyu Xie ${ }^{* a}$ and Bingqing Wei (D) ${ }^{b}$
}

\begin{abstract}
Sluggish dynamics of polysulfide (LiPS) conversion leads to reduced utilization of active sulfur and rapid capacity decay. Introducing catalysts into lithium-sulfur battery systems is a feasible and imperative strategy to tackle this problem. Previous research studies have mainly been focused on selecting new catalysts and design functional structures to improve performance, and ignoring the interaction between catalysts and their carriers. Herein, by simply fabricating a high-efficiency ZnS quantum dotagraphene nanosheet catalyst (ZnS QD@rGO), we utilized enhanced interfacial electronic interaction to accelerate polysulfide conversion for high energy density Li-S batteries. With the smaller size of ZnS, the interfacial electronic interaction becomes more enhanced, which was evidenced by DFT calculations and XPS experiments. After mixing with sulfur, the electrodes achieved a high capacity of $857.8 \mathrm{~mA} \mathrm{~h} \mathrm{~g}^{-1}$ at $1 \mathrm{C}$ and a retention of $91.2 \%$ after 300 cycles. Also, a sulfur cathode with a high actual capacity of $\sim 4.0 \mathrm{~mA} \mathrm{~h} \mathrm{~cm}{ }^{-2}$ could be obtained, with no obvious capacity decay within 100 cycles. We believe that this strategy represents a new perspective on designing efficient high-load electrodes for $\mathrm{Li}-\mathrm{S}$ batteries.
\end{abstract}

Received 26th March 2020

Accepted 15th May 2020

DOI: $10.1039 / \mathrm{d} 0 \mathrm{nr} 02429 \mathrm{e}$

rsc.li/nanoscale cycling performance. ${ }^{8}$ Researchers have done plenty of work on introducing porous carbon as a sulfur carrier, however, the weak bonding between carbon materials and LiPS cannot unlock the full potential of lithium-sulfur batteries. ${ }^{9}$ Therefore, new approaches are needed for the advanced sulfur cathode in terms of increased electrochemical dynamics, confined polysulfides and high sulfur loading. ${ }^{10,11}$

One of the most feasible topics in enhancing the conversion kinetics of Li-S batteries is to introduce catalytically active materials into battery systems, ${ }^{6,8,12,13}$ Some polar materials such as $2 \mathrm{~d}$ metal dichalcogenides and oxides have been widely investigated in Li/Na-S batteries owing to their chemisorption with LiPS and strong catalysis activity. ${ }^{14-16}$ Lee et al. acquired Li-S batteries with increased rate performance and cycling stability by employing sulfur-deficient $\mathrm{MoS}_{2}$ nanoflakes. In their work, a large number of sulfur deficiencies were introduced into the $\mathrm{MoS}_{2}$ nanoflakes, which work as catalytic centers to catalyse fast conversion of soluble polysulfides in the batteries. ${ }^{17}$ However, defect control or tailoring is not enough to maximize the catalytic efficiency. Recently, rationally designing efficient heterogeneous/homogeneous structures emerges to be a promising method to construct highenergy-density metal-sulfur batteries. ${ }^{18}$ Zhang et al. demonstrated a hybrid structure of the $\mathrm{CoS}_{2}-\mathrm{rGO}$ composite that electrochemically accelerated the redox reactions of polysulfides. ${ }^{19}$ Qiao et al. fabricated a two-dimensional heterostructured 
MoN-VN for new model sulfur cost. They evidenced that the electronic structure of MoN can be tailored by the incorporation of $\mathrm{V}$, leading to enhanced polysulfide adsorption. ${ }^{20}$

In fact, a catalyst is not independent of the system but exists as part of heterostructures with other conductive carriers such as carbon materials or polymers. ${ }^{21-23}$ Thus, the performance of a catalyst is also related to the interaction between catalysts and carriers. It has been previously reported that the surface chemistry and local electron state of the sulfur cathode materials is essential for investigating the polysulfide adsorption behaviors in sodium-sulfur batteries. ${ }^{24}$ In our hypothesis, when the carrier and its loaded catalysts have different bandgap energies and contacts, electron migration may occur and form an electron-rich/deficient surface, resulting in an internal electric field at the interface..$^{25,26}$ During the oxidation/ reduction reaction, the reactant will be attracted to the electron-rich/deficient surface, which may supply sufficient raw materials to ensure continuous reaction progress. Besides, due to the spontaneous electron migration, charge migration will be greatly enhanced, thus accelerating the conversion process. $^{26-28}$ The interfacial electronic interaction mentioned earlier exists in all the contacts between catalysts and carriers, but the influence on enhancing the catalysis activity is not obvious due to the large size of catalysts and the large area of the non-polar interface. ${ }^{26,29}$ Downsizing the size of catalysts will be a simple and feasible way to achieve a highly active catalyst.

In this work, we suggest a new strategy to construct heterostructure composites as the cathode by synthesizing a $\mathrm{ZnS}$ quantum dot@graphene nanosheet (ZnS QD@rGO) through a simple approach for high energy density Li-S batteries, as is shown in Fig. 1a. The charge distribution calculation by density functional theory (DFT) shows that minimizing the size of heterostructures can create the polarized surface through the electronic interactions, which can be evidenced by elemental analysis via the X-ray photoelectron spectroscopy (XPS) test. It can be proved by the visual adsorption test and electrochemical analysis that nanoheterostructures of $\mathrm{ZnS}$ QD@rGO and the interfacial electronic interactions play an

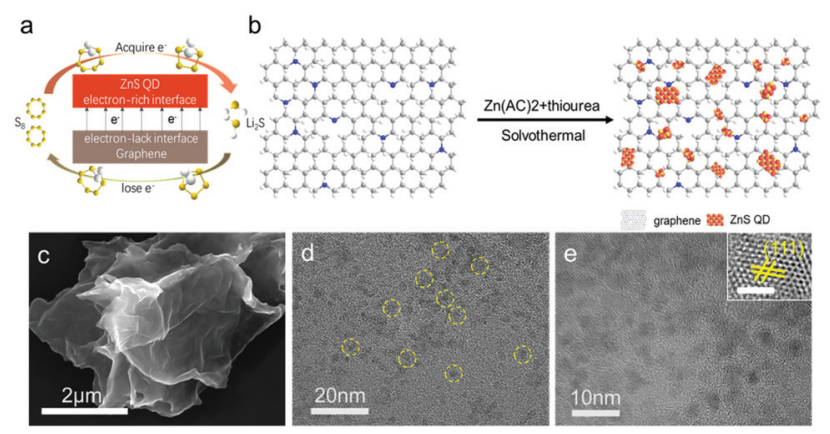

Fig. 1 (a) Schematic of the synthesis process of ZnS QDarGO. (b) Schematic representation of the Li-S ZnS QDarGO contained cathode. (c) SEM image of ZnS QDarGO samples. (d) TEM image of ZnS QDarGO samples (yellow cycles represent the size of ZnS QDs. (e) HR-TEM image of ZnS QDarGO (inset scale bar $=2 \mathrm{~nm}$ ). important role in enhancing the charge transport, strengthening binding with LiPSs and accelerating dynamics for fast redox reaction. Benefiting from the unique structure of $\mathrm{ZnS}$ QD@rGO materials, the electrode with ZnS QD@rGO delivers a high-rate capability of about 1062 and $500 \mathrm{~mA} \mathrm{~h} \mathrm{~g}^{-1}$ at rates of 0.2 and $5 \mathrm{C}\left(1 \mathrm{C}=1675 \mathrm{~mA} \mathrm{~h} \mathrm{~g}^{-1}\right)$, respectively. As a consequence, the battery displays a desirable capacity retention of $91.2 \%$ over more than 300 cycles at 1 C. By increasing the sulfur loading of the electrode, a cathode with an actual capacity of $\sim 4.0 \mathrm{~mA} \mathrm{~h} \mathrm{~cm} \mathrm{c}^{-2}$ can also be obtained.

\section{Experimental}

\section{Synthesis of ZnS QD@rGO, ZnS NT@rGO, and ZnS QDs}

All the chemicals were bought from Sigma-Aldrich under analytical grade without further purification. As is shown in Fig. 1b, we prepared ZnS QD@rGO samples through a simple one-step hydrothermal method. ${ }^{25}$ The transmission electron microscopy (TEM) and scanning electron microscopy (SEM) images of the as-obtained graphene are shown in Fig. S1. $\dagger$ Firstly, graphene oxide (GO) was synthesized through the modified Hummers' method, as reported before. Then, in a typical fabrication process, $60 \mathrm{mg}$ of the as-prepared GO powder was dispersed in $70 \mathrm{ml}$ deionized water and dispersed well through stirring and ultrasound. After that, $300 \mathrm{mg}$ thiourea $\left(\mathrm{CH}_{4} \mathrm{~N}_{2} \mathrm{~S}\right), 280 \mathrm{mg} \mathrm{Zn}(\mathrm{AC})_{2}\left(\mathrm{CH}_{6} \mathrm{O}_{4} \mathrm{Zn} \cdot 2 \mathrm{H}_{2} \mathrm{O}\right)$, and $100 \mathrm{mg}$ poly(acrylic acid) (PAA, used as forming ZnS QD sites) were added into the mixture. After mechanically stirring using magnets for one night, the solution was transferred to a Teflon-lined autoclave at $180{ }^{\circ} \mathrm{C}$ for 24 hours. Then, the obtained compound was washed with deionized water and ethanol several times. Finally, the as-obtained solid was dried and heated at $400{ }^{\circ} \mathrm{C}$ for 5 hours under a nitrogen atmosphere. For comparison, a pure ZnS QD sample was prepared without adding GO, while ZnS NT@rGO was prepared without PAA through a similar method to ZnS QD@rGO.

\section{Characterization}

SEM (FEI Tecnai G2 F30), TEM (Talos-F200X), X-ray diffraction (XRD) (X'Pert PRO, Cu K $\alpha$ ), XPS (ESCALAB 250xi), Raman (inVia confocal Raman microscope with a $532 \mathrm{~nm}$ laser), and Brunauer-Emmett-Teller area (BET, ASAP 2020 surface area analyser) were used to characterize the morphology and structure of ZnS QDs and ZnS NT samples. Thermogravimetric analysis (TGA, METTLER TOLEDO) was used to certify the ZnS amount of ZnS QD@rGO samples under an argon atmosphere from room temperature to $900{ }^{\circ} \mathrm{C}$ with a heating rate of $10^{\circ} \mathrm{C}$ $\min ^{-1}$.

\section{First-principles simulations}

Interface charge distribution between ZnS QD and rGO was calculated by using DFT through generalized gradient approximation (GGA). The CASTEP code and Perdew-Burke-Ernzerhof (PBE) exchange-correlation parameterization were applied as reported before. $^{22}$ The cut-off voltage of $450 \mathrm{eV}$ was employed 
when the plane-wave expanded from all the cut-offs specific to each element in this system. Also, the $(1 \times 1 \times 1) k$-point grid was performed to simulate the distribution of atoms. The interaction between ZnS QDs and rGO was simulated using DFT semi-core pseudopods, with a total energy convergence of $10^{-5} \mathrm{eV}$. According to the XRD and TEM data, the (111) crystal plane occupies the main exposed crystal surface in ZnS QD samples. Here, for simplifying calculations, the (111) plane was used as an active surface of $\mathrm{ZnS}$ crystals. Also, in this model, only $\mathrm{N}$ was chosen as the doping element in graphene. The lattice parameters of cubic ZnS are $a=b=c=0.541$ in this model.

\section{Electrochemical measurements}

For electrode fabrication with $1 \mathrm{mg} \mathrm{cm}^{-2}$ sulfur loading, firstly, sulfur, sample (ZnS QD@rGO, ZnS NT@rGO, and rGO powders), acetylene black and PVDF were ground for 1 hour with a mass ratio of $0.56: 0.14: 0.2: 0.1$. Then, the mixture was added to N-methyl pyrrolidone (NMP) solution with $21 \%$ solid content and stirred overnight to form a slurry. After that, the slurry was cast on carbon coated $\mathrm{Al}$ foil and dried under vacuum at $50{ }^{\circ} \mathrm{C}$ for $12 \mathrm{~h}$. The high sulfur loading electrode was prepared similarly by changing the mass ratio to $0.48: 0.12: 0.28: 0.12$ and the solid content to $23 \%$. The electrochemical performances were tested using CR2016 cointype cells consisting of a $12 \mathrm{~mm}$ diameter sulfur containing working electrode, separator (Celgard 2500 in $19 \mathrm{~mm}$ diameter), and lithium foil (16 $\mathrm{mm}$ in diameter and $0.45 \mathrm{~mm}$ in thickness). The electrolyte was prepared using $1 \mathrm{M}$ lithium bistrifluoromethane-sulfonylimide (LiTFSI) in 1,2-dimethoxyethane (DME) and 1,3-dioxolane (DOL) $(1: 1, \mathrm{v} / \mathrm{v})$ with $2 \mathrm{wt} \%$ $\mathrm{LiNO}_{3}$ additive. The amount of electrolyte is $8 \mu \mathrm{l} \mathrm{mg}^{-1}$ based on the mass of sulfur in the electrode. The cell was assembled in the glove box filled with highly-pure argon $\left(\mathrm{O}_{2}, \mathrm{H}_{2} \mathrm{O}<\right.$ $0.1 \mathrm{ppm})$. Cycling and rate performance of the batteries were performed using a LAND CT2001A battery tester at the voltage range of 1.7 to $2.8 \mathrm{~V}$ ( $v s$. $\mathrm{Li}^{+} / \mathrm{Li}$ ). The $\mathrm{CV}$ curves were collected on an electrochemical workstation (Solartron Analytical 1400E) at the voltage range of 1.7 to $2.8 \mathrm{~V}$. EIS was measured in the frequency range of $100 \mathrm{kHz}$ to $0.01 \mathrm{~Hz}$.

\section{Results and discussion}

As is shown in Fig. 1b, the ZnS QD@rGO sample was synthesized through a simple solvothermal reaction at $180{ }^{\circ} \mathrm{C}$ using PAA, $\mathrm{Zn}(\mathrm{ac})_{2}$ and thiourea. In the synthesis of $\mathrm{ZnS}$ quantum dots, usually, the metal ions can be homogeneously dispersed into the GO matrix through its carboxylic acid groups. However, the carboxylic acid functional groups are mainly located at the graphene edge, and most of them will be removed after reduction. PAA plays an improtant role in inhibiting the growth of $\mathrm{ZnS}$ crystals through avoiding uniting of atoms, when it was introduced into the reaction system, which possesses an abundance of carboxylic acid functional groups. Thus, the interactions between $\mathrm{ZnS} \mathrm{QD}$ and graphene are mostly van der Waals contact between two phases rather than chemical bonds. ${ }^{30}$ As a proof, as is shown in Fig. S2, $\uparrow$ the C1s and N1s peaks in ZnS QD@rGO samples demonstrate no extra bonding with ZnS QDs such as Zn-C or Zn-N. As a control, the ZnS nanosheet@graphene nanosheet (ZnS NT@rGO) was also fabricated by a similar method without the introduction of PAA. Graphene was reduced directly from GO powder via the solvothermal reaction. When used as catalysts in $\mathrm{Li}-\mathrm{S}$ batteries, the interfacial electronic interaction between $\mathrm{ZnS}$ and graphene would be of vital importance in adsorbing LiPSs and enhancing the conversion kinetics (Fig. 1a).

As can be seen in Fig. 1c, no obvious nanosheets could be observed on the rGO layer, while element mapping (Fig. S3 $\dagger$ ) shows plenty of zinc element and sulfur element existing on the graphene sheet, implying high dispersion of ZnS QDs on the graphene nanosheet. In contrast, large area $\mathrm{ZnS}$ nanosheets can be found on the surface of graphene in the ZnS NT@rGO sample; meanwhile, zinc and sulfur patterns on EDS mapping images show severe aggregation. Based on the TEM images in Fig. 1d, highly dispersed ZnS QDs can be seen on the graphene sheet. The HR-TEM image (Fig. 1e) of ZnS QDs/N-rGO shows the uniform size of ZnS QDs ( $\sim 5 \mathrm{~nm})$ and the (111) plane lattice spacing of the cubic ZnS crystal.

The structure and composition information of the obtained ZnS QD@rGO (Fig. 2) and ZnS NT@rGO samples (Fig. S5 $\dagger$ ) were analyzed by Raman, XRD, BET, and TGA. Both samples show similar Raman spectra (Fig. 2a and Fig. S5a $\dagger$ ). The ratio of $I_{\mathrm{D}} / I_{\mathrm{G}}$ is $\sim 1.00$, demonstrating the well-graphitized status of rGO substrates. ${ }^{31,32}$ The X-ray diffraction (XRD) patterns (Fig. 2b) reveal the (111), (220) and (311) crystal planes, which well-match the face centered cubic ZnS (JCPDS\# 05-0566), whereas the ZnS NT@rGO sample exhibits many different crystal planes (Fig. S5b $\dagger$ ). As is shown in Fig. 2c, ZnS QD@rGO exhibits a specific BET surface area of $63.1 \mathrm{~m}^{2} \mathrm{~g}^{-1}$, which is much larger than that of ZnS NT@-rGO $\left(31.3 \mathrm{~m}^{2} \mathrm{~g}^{-1}\right.$, Fig. S5d $\dagger$ ). The small size of quantum dots provides a larger
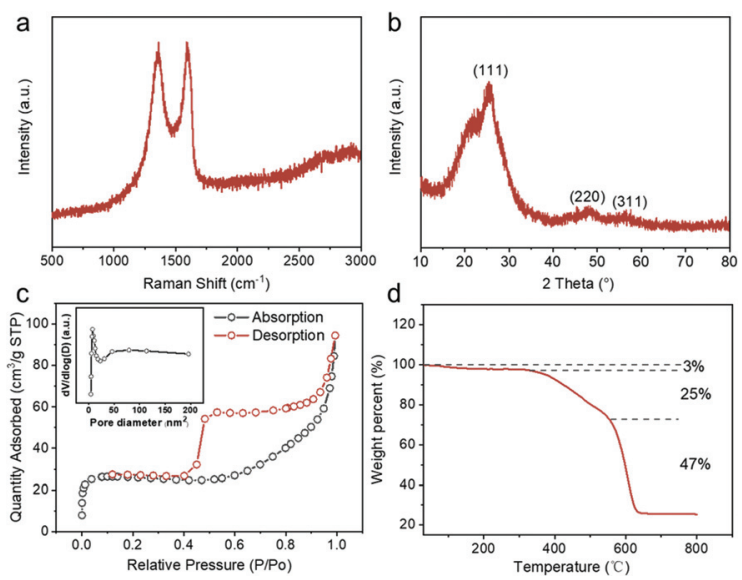

Fig. 2 Raman pattern (a), XRD pattern (b), BET surface area (c) (pore size distribution inset), and TGA curve of the as-synthesized ZnS QDarGO (d). 
reaction area, to the benefit of accelerating the reaction rate. ${ }^{33,34}$ Furthermore, from the TGA curves in Fig. $2 d$ and Fig. S5c, $\dagger$ it was observed that the main difference between the $\mathrm{ZnS}$ QD@rGO sample and ZnS NT@rGO sample is a specific area, which primarily resulted from the smaller size and homogeneous dispersion of $\mathrm{ZnS}$ quantum dots.

The adsorption ability of LiPSs and electrochemical catalysis properties of the ZnS QD@rGO samples are shown in Fig. 3. The strong binding between hosts and LiPSs provides plenty of ingredients for further conversion which is found to be one of the dominant factors for the Li-S battery charge-discharge process. Herein, $5 \mathrm{mM} \mathrm{Li}_{2} \mathrm{~S}_{6}$ in DOL/DME (V:V = 1:1) was employed as the representative LiPS. ZnS QD@rGO, ZnS NT@rGO, and graphene with the same weight were added into the LiPS solution. After $6 \mathrm{~h}$, the color of the $\mathrm{Li}_{2} \mathrm{~S}_{6}$ solution with ZnS QD@rGO is much lighter than other samples, indicating the enhanced adsorption of ZnS QD@rGO with the polysulfide. In Fig. 3b, the UV-vis spectra of these supernatant solutions after absorption further show a reduced LiPS concentration of the ZnS QD@rGO sample.

To further verify the electrocatalysis process of the $\mathrm{ZnS}$ QD@rGO samples in LiPS transformation, symmetrical coin cells with no sulfur in the electrode were constructed using a $\mathrm{Li}_{2} \mathrm{~S}_{6}$-containing electrolyte, in which the concentration of $\mathrm{Li}_{2} \mathrm{~S}_{6}$ solution is $0.5 \mathrm{M}$ (Fig. 3c). Cyclic voltammetry (CV) curves of all candidates were measured from $-0.7 \mathrm{~V}$ to $0.7 \mathrm{~V}$ with a scan rate of $10 \mathrm{mV} \mathrm{s}^{-1}$. The polarization patterns are mainly contributed by the $\mathrm{Li}_{2} \mathrm{~S}_{6}$ redox current, while the contribution from the capacitive current can be neglected. The $\mathrm{ZnS}$ QD@rGO symmetric cell exhibits the highest polarization current, demonstrating the better catalytic performance provided by the ZnS QD@rGO samples compared with the nanosheet counterpart. ${ }^{6,35}$ The interfacial charge redistribution of the heterostructures as well as more active sites in the ZnS QD nanostructure, which will cause the increase in the electrochemical active area, thus increase the catalytic activity of ZnS QD@rGO samples in lithium-sulfur batteries. ${ }^{36}$

We also evaluated the polysulfide redox chemistry by analyzing the CV scans. During the discharge process, sulfur is reduced to soluble LiPSs first, and then to solid $\mathrm{Li}_{2} \mathrm{~S}_{2}$ or $\mathrm{Li}_{2} \mathrm{~S}$. After that, $\mathrm{Li}_{2} \mathrm{~S}$ is oxidized back to elemental sulfur during the charging process. The round trip process faces a serious problem with the loss of active materials resulting from poor kinetics. In Fig. 3d, the two cathodic peaks at 2.25-2.40 and

\section{a}
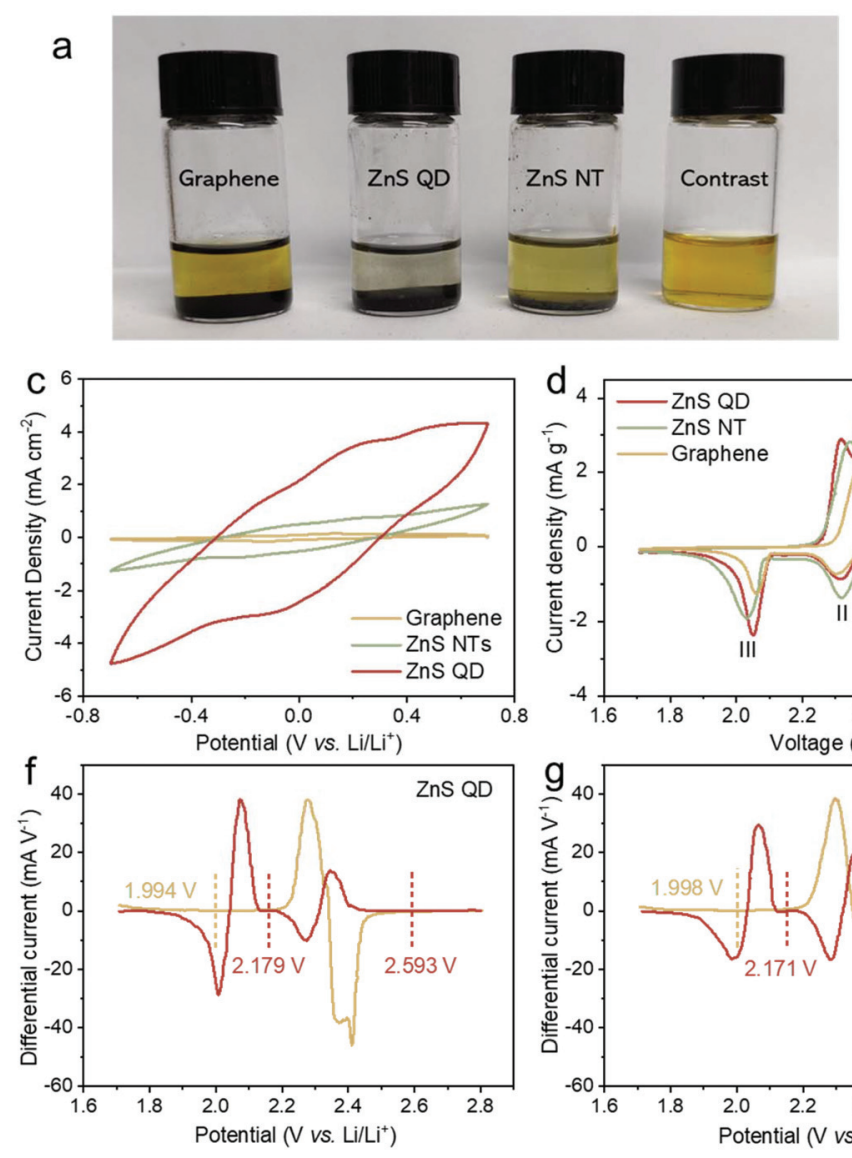
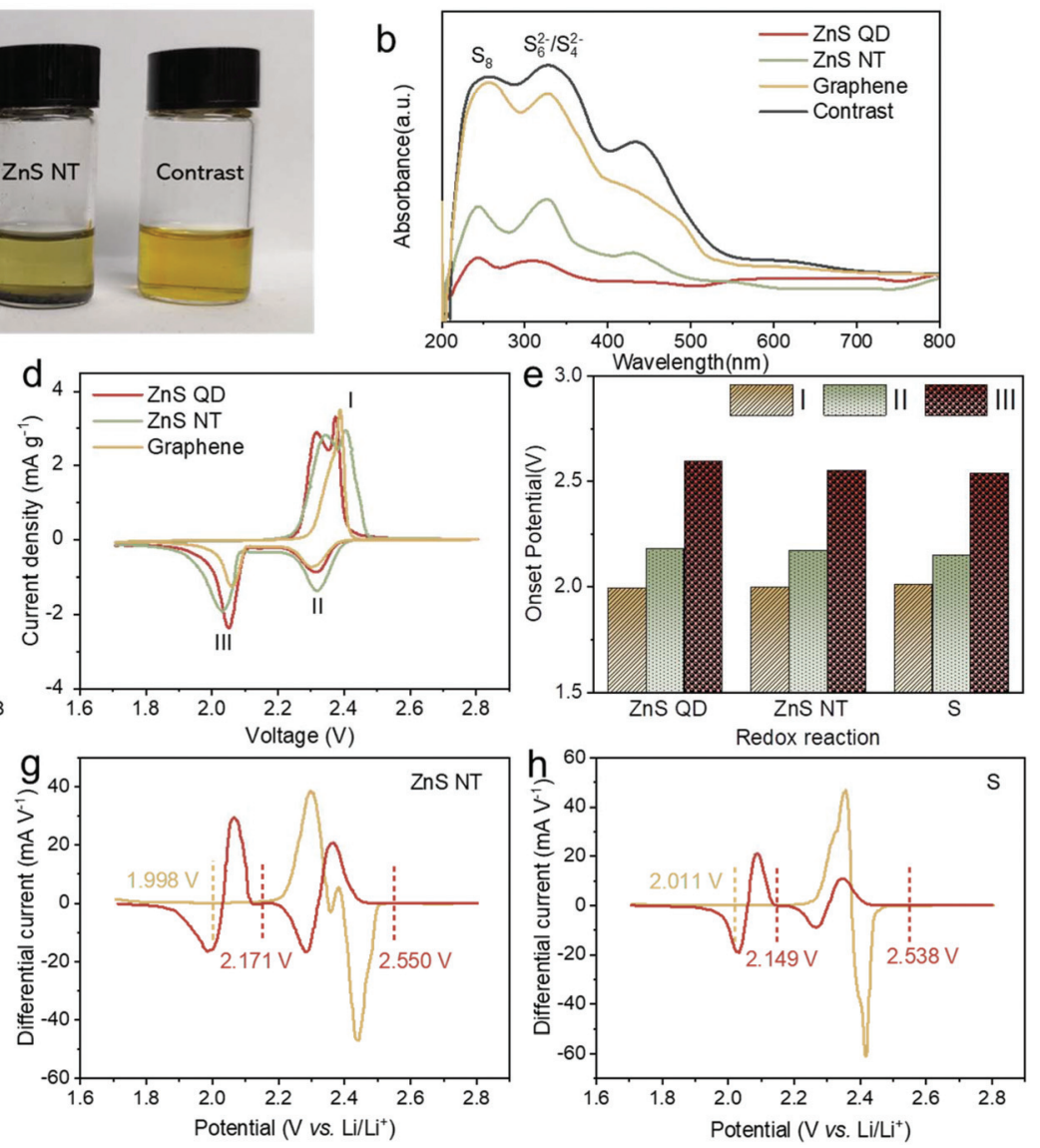

Fig. 3 (a) LiPS $\left(\mathrm{Li}_{2} \mathrm{~S}_{6}\right)$ adsorption test using graphene, ZnS QDarGO, and ZnS NTarGO as adsorbents, (b) corresponding UV-vis spectra of the supernatant solutions. (c) $\mathrm{CV}$ curve of symmetrical $\mathrm{Li}_{2} \mathrm{~S}_{6}-\mathrm{Li}_{2} \mathrm{~S}_{6}$ cells from -0.7 to $0.7 \mathrm{~V}$, the sweep rate is $10 \mathrm{mV} \mathrm{s}{ }^{-1}$. (d) $\mathrm{CV}$ profiles of lithium-sulfur batteries with ZnS QD@rGO, ZnS NT@rGO, and graphene additives. (e) Onset potentials of asymmetrical Li-S cells corresponding in ( $\mathrm{f}-\mathrm{h}$ ). Differential CV curves of lithium-sulfur batteries with ZnS QD@arGO (f), ZnS NTarGO (g), and graphene additives (h), respectively. 
1.90-2.10 V can be attributed to the two processes of S8 reduction ( $\mathrm{S} 8$ to LiPSs to $\mathrm{Li}_{2} \mathrm{~S}_{2} / \mathrm{Li}_{2} \mathrm{~S}$ ). The two subsequent anodic peaks at 2.25-2.60 $\mathrm{V}$ correspond to the complex oxidation process from $\mathrm{Li}_{2} \mathrm{~S}$ to elemental sulfur. ${ }^{37,38}$ Compared with the ZnS NT@rGO sample, the ZnS QD@rGO sample results in a significantly reduced voltage gap (0.32 V vs. $0.4 \mathrm{~V})$, revealing the promoted reversibility of redox reactions for robust Li-S batteries. For further analyzing the CV curves, the onset potential of each cell was calculated based on difference curves (Fig. 3f-h). As listed in Fig. 3e, the battery based on ZnS QD@rGO shows an earlier start of oxidation and reduction peaks than other samples, which can be attributed to the catalytic activity of ZnS QDs.

The possible reason can be ascribed to interfacial charge redistribution. As is shown in Fig. $4 \mathrm{a}$, the Fermi level $\left(E_{\mathrm{F}}\right)$ of graphene is $-4.6 \mathrm{eV}$ from the vacuum electron level $\left(E_{\mathrm{vac}}\right)$, which is much higher than that of $\mathrm{ZnS}\left(E_{\mathrm{F}}=-7 \mathrm{eV}\right) \cdot{ }^{39,40}$ According to the DFT calculation, as is shown in Fig. 4c and d, driven by the large potential energy difference, electrons spontaneously slide from graphene to $\mathrm{ZnS}$ at the interface when the two materials come into contact, leading to the presence of an internal electric field. ${ }^{26,41}$ This phenomenon can be accounted for the strong interfacial polarization between the two materials. As a result, the electrons (yellow) gathered at $\mathrm{ZnS}$ QDs with high density while holes (blue) are collected at the nitrogen doped rGO, indicating the separation and redistribution of the electron-hole pairs.

The XPS patterns of ZnS QD@rGO, ZnS NT@rGO, and ZnS QDs were used to prove the hypothesis of electron redistribution at the interface. The Zn 2p peak of the ZnS QD@rGO sample shows an obvious blueshift phenomenon compared with other counterparts, which can be interpreted as intense polarization and electric field at the interface between ZnS QD and rGO resulting from interfacial electron migration. By con-
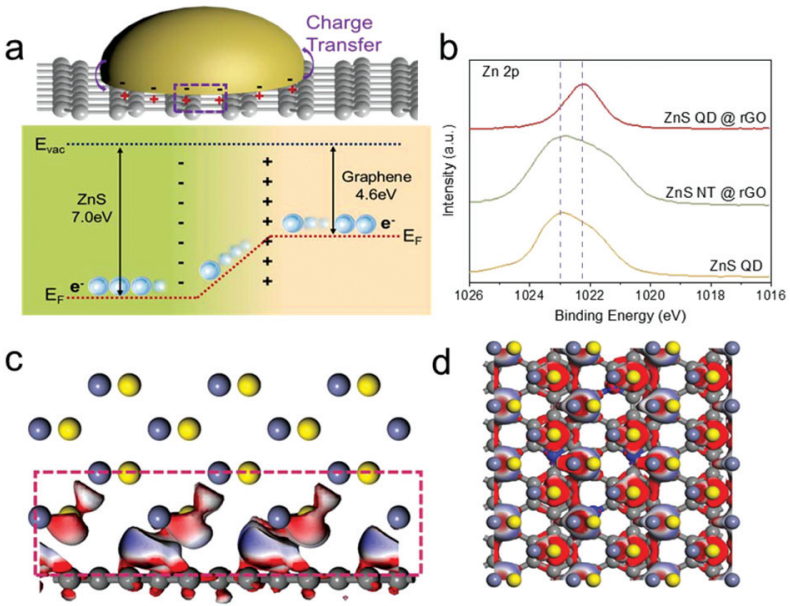

Fig. 4 (a) Schematic demonstration of the electron distribution at the interface between graphene and ZnS. (b) Zn $2 p$ peaks of the XPS pattern of ZnS QDarGO, ZnS NTarGO, and pure ZnS QDs. (c and d) The charge distribution calculated by density functional theory at the interface of ZnS QDs and N-rGO from the top (c) and side views (d). trast, the ZnS NT@rGO sample shows no peak shift, meaning that the as-mentioned interfacial charge redistribution will be noticeable and therefore change the catalyzing process only when the size of the nanoparticle is small enough. This phenomenon can be easily demonstrated by catalytic results Fig. 5a shows the Electrochemical Impedance Spectroscopy (EIS) values of Li-S batteries with ZnS QD@rGO, ZnS NT@rGO, and graphene additives. The ZnS QD@rGO cathode presents a lower charge-transfer resistance due to the strongest interaction between LiPSs and ZnS QD@rGO. Fig. 5e shows the galvanostatic discharge/charge (GDC) profiles of the first cycle of the three electrodes. The first plateau during the discharge process at $\sim 2.35 \mathrm{~V}$ can be attributed to the conversion from $\mathrm{S}_{8}$ to $\mathrm{Li}_{2} \mathrm{~S}_{8}$. The voltage corresponding to the second discharge plateau can be classified as the reduction from $\mathrm{Li}_{2} \mathrm{~S}_{4}$ to $\mathrm{Li}_{2} \mathrm{~S}_{2}$ and $\mathrm{Li}_{2} \mathrm{~S}$. The specific values are $\sim 2.10 \mathrm{~V}$ for $\mathrm{ZnS}$ QD@rGO and $2.05 \mathrm{~V}$ for other samples. The smaller bandgap of the ZnS QD@rGO sample induces lower polarization and a better electrocatalysis activity of LiPSs, contributing to stable long-term cycling performance. The comparison of cycling performance is shown in Fig. S6a. $\dagger \mathrm{ZnS}$ QD@rGo and $\mathrm{ZnS}$ NT@rGO cathodes deliver a similar capacity $\left(\sim 1100 \mathrm{~mA} \mathrm{~h} \mathrm{~g}{ }^{-1}\right)$ at first. In contrast, after 100 cycles, the ZnS QD@rGO cathode shows a more stable cycling ability than the ZnS QD@rGO cathode $(74.6 \%$ vs. $46.7 \%)$ with higher coulombic efficiency (99\% vs. 96\%), demonstrating high round trip efficiency and improved conversion dynamics of LiPSs. We further measured the CV curves of the ZnS QD@rGO cathode at the current density of $0.1 \mathrm{mV} \mathrm{s}^{-1}$ (Fig. S5b†) and found nearly coinciding
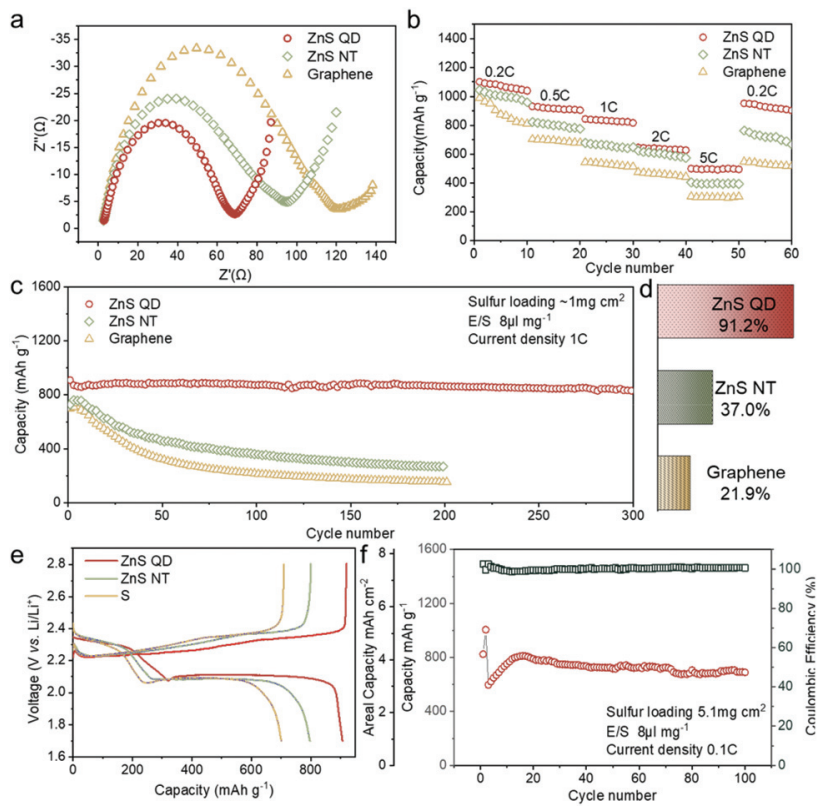

Fig. 5 Detailed Li-S battery performances: (a) EIS results of the battery based on ZnS QDarGO, ZnS NTarGO, and graphene cathodes before cycles. (b) Rate performance of the batteries. (c) Long cycling performance at $1 \mathrm{C}$. (d) Corresponding capacity retention ratio based on cycling performance in (c). (e) 1st cycle charge-discharge curves in (c). (f) Cycling performance based on high actual sulfur loadings $\left(\sim 5 \mathrm{mg} \mathrm{cm}^{-2}\right)$. 
curves of the first several cycles. This high consistency implies minimal polysulfide shuttle, proving the high cycling stability and constant electrode structure during charge and discharge processes.

Furthermore, long-term cycling stability, rate capacity, and high area capacity performance are provided in Fig. $5 \mathrm{c}-\mathrm{f}$. The long-life cycling properties of the lithium-sulfur battery with the ZnS QD@rGO cathode are measured at the current density of $1 \mathrm{C}$ (Fig. 5c). It exhibits an initial discharge capacity of $857.8 \mathrm{~mA} \mathrm{~h} \mathrm{~g}^{-1}$. After the 300 discharging-charging cycle test at $1 \mathrm{C}$, the capacity retention rate is $91.2 \%$, displaying a stable long-term cycling performance. To assess the ability of fast charging and discharging, rate performance was first performed from 0.2 to $5 \mathrm{C}$ (Fig. 5b). The ZnS QD@rGO cathode delivered a specific capacity of $1062.2 \mathrm{~mA} \mathrm{~h} \mathrm{~g}^{-1}$ at $0.2 \mathrm{C}$, and the battery showed 932.1, 845.4,645.9, and $499.4 \mathrm{~mA} \mathrm{~h} \mathrm{~g}^{-1}$ at current densities of $0.5,1,2$, and $5 \mathrm{C}$, respectively, which are much higher than those of the ZnS NT@rGO cathode and graphene cathode, especially at high current densities. In addition, when the current density skipped back to $0.2 \mathrm{C}$, almost all of the capacities were recovered, but the $\mathrm{ZnS}$ QD@rGO cathode demonstrates the highest initial capacity retention of $0.2 \mathrm{C}$, demonstrating excellent capacity holding ability and electrode structure after high current density.

In consideration of practical use, we increased the sulfur

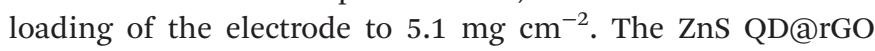
cathode was first activated at $0.05 \mathrm{C}$, and then tested at $0.1 \mathrm{C}$ with a low $\mathrm{E} / \mathrm{S}$ ratio of $8 \mu \mathrm{l} \mathrm{mg}^{-1}$. The cathode demonstrated an outstanding initial capacity of $1011 \mathrm{~mA} \mathrm{~h} \mathrm{~g}^{-1}$ and could retain a high specific capacity of $\sim 800 \mathrm{~mA} \mathrm{~h} \mathrm{~g}^{-1}$ at $0.1 \mathrm{C}$ (Fig. 5f). The calculated actual capacity of the ZnS QD@rGO cathode can obtain up to $\sim 4.0 \mathrm{~mA} \mathrm{~h} \mathrm{~cm}{ }^{-2}$ after 100 cycles at the current density of $0.1 \mathrm{C}$, which is close to the value of the state-of-art commercial cathode materials of Li-ion batteries.

\section{Conclusions}

In conclusion, interfacial electronic interaction is revealed by DFT calculations and applied in the LiPS conversion process. By using ZnS QD@rGO as the LiPS promotor, the polarized surface was created through the enhanced interfacial electronic interactions, which have been evidenced by XPS elemental analysis. Benefiting from the enhanced catalysis effect of the as-fabricated heterostructure, the electrode with $\mathrm{ZnS}$ QD@rGO delivered excellent cycling stability ( $91.2 \%$ capacity retention after 300 cycles at $1 \mathrm{C}$ ) and rate performance $\left(500 \mathrm{~mA} \mathrm{~h} \mathrm{~g}{ }^{-1}\right.$ at $\left.5 \mathrm{C}\right)$. Also, a sulfur cathode with an area capacity of $4.0 \mathrm{~mA} \mathrm{~h} \mathrm{~cm}{ }^{-2}$ was also acquired at $0.1 \mathrm{C}$, which makes this strategy more practicable for commercial use. We expect that it can provide new additions on rational designing catalysts for metal-sulfur batteries.

\section{Conflicts of interest}

There are no conflicts to declare.

\section{Acknowledgements}

The authors acknowledge the financial support provided by the National Natural Science Foundation of China (51974256), the Outstanding Young Scholars of Shaanxi (2019JC-12), the Natural Science Basic Research Plan in Shaanxi Province of China (2019JLZ-01, 2019JLM-29), the Fundamental Research Funds for the Central Universities (19GH020302 and 3102019JC005), the State Key Laboratory of Solidification Processing (NPU), China (2019-TS-06), and the China Postdoctoral Science Foundation (2018M641015). Zhang Kun was supported by the China Scholarship Council for 2 year study at the National University of Singapore.

\section{References}

1 A. Manthiram, Y. Fu, S.-H. Chung, C. Zu and Y.-S. Su, Chem. Rev., 2014, 114, 11751.

2 S. Bai, X. Liu, K. Zhu, S. Wu and H. Zhou, Nat. Energy, 2016, 1, 16094.

3 X. Huang, J. Tang, B. Luo, R. Knibbe, T. Lin, H. Hu, M. Rana, Y. Hu, X. Zhu, Q. Gu, D. Wang and L. Wang, Adv. Energy Mater., 2019, 1901872, DOI: 10.1002/aenm.201901872.

4 L. Xu, H. Zhao, M. Sun, B. Huang, J. Wang, J. Xia, N. Li, D. Yin, M. Luo, F. Luo, Y. Du and C. Yan, Angew. Chem., Int. Ed., 2019, 58, 11491.

5 K. Zhang, K. Xie, K. Yuan, W. Lu, S. Hu, W. Wei, M. Bai and C. Shen, J. Mater. Chem. A, 2017, 5, 7309-7315.

6 K. Zhang, Z. Chen, R. Ning, S. Xi, W. Tang, Y. Du, C. Liu, Z. Ren, X. Chi, M. Bai, C. Shen, X. Li, X. Wang, X. Zhao, K. Leng, S. J. Pennycook, H. Li, H. Xu, K. P. Loh and K. Xie, ACS Appl. Mater. Interfaces, 2019, 11, 25147.

7 S.-H. Chung, L. Luo and A. Manthiram, ACS Energy Lett., 2018, 3, 568.

8 T.-G. Jeong, D. S. Choi, H. Song, J. Choi, S.-A. Park, S. H. Oh, H. Kim, Y. Jung and Y.-T. Kim, ACS Energy Lett., 2017, 2, 327.

9 J. Ren, Y. Zhou, H. Wu, F. Xie, C. Xu and D. Lin, J. Energy Chem., 2019, 30, 121.

10 S.-Y. Li, W.-P. Wang, H. Duan and Y.-G. Guo, J. Energy Chem., 2018, 27, 1555.

11 H. Li, D. Chao, B. Chen, X. Chen, C. Chuah, Y. Tang, Y. Jiao, M. Jaroniec and S. Z. Qiao, J. Am. Chem. Soc., 2020, 142, 2012.

12 L. Luo and A. Manthiram, ACS Energy Lett., 2017, 2, 2205.

13 S. B. Patil, H. J. Kim, H.-K. Lim, S. M. Oh, J. Kim, J. Shin, H. Kim, J. W. Choi and S.-J. Hwang, ACS Energy Lett., 2018, 3, 412 .

14 M. Salama, Rosy, R. Attias, R. Yemini, Y. Gofer, D. Aurbach and M. Noked, ACS Energy Lett., 2019, 436-446, DOI: 10.1021/acsenergylett.8b02212.

15 Z. Xiao, Z. Yang, L. Zhang, H. Pan and R. Wang, ACS Nano, 2017, 11, 8488.

16 C. Ye, D. Chao, J. Shan, H. Li, K. Davey and S.-Z. Qiao, Matter, 2020, 2, 323. 
17 H. Lin, L. Yang, X. Jiang, G. Li, T. Zhang, Q. Yao, G. W. Zheng and J. Y. Lee, Energy Environ. Sci., 2017, 10, 1476. 18 Z.-W. Zhang, H.-J. Peng, M. Zhao and J.-Q. Huang, Adv. Funct. Mater., 2018, 28, 1707536.

19 Z. Yuan, H. J. Peng, T. Z. Hou, J. Q. Huang, C. M. Chen, D. W. Wang, X. B. Cheng, F. Wei and Q. Zhang, Nano Lett., 2016, 16, 519.

20 C. Ye, Y. Jiao, H. Jin, A. D. Slattery, K. Davey, H. Wang and S. Z. Qiao, Angew. Chem., Int. Ed., 2018, 57, 16703.

21 L. Tan, X. Li, Z. Wang, H. Guo and J. Wang, ACS Appl. Mater. Interfaces, 2018, 10, 3707.

22 L. Kong, X. Chen, B. Q. Li, H. J. Peng, J. Q. Huang, J. Xie and Q. Zhang, Adv. Mater., 2018, 30, 1705219.

23 T. Lei, W. Chen, J. Huang, C. Yan, H. Sun, C. Wang, W. Zhang, Y. Li and J. Xiong, Adv. Energy Mater., 2017, 7, 1601843.

24 P. Wang, T. Jiang, C. Zhu, Y. Zhai, D. Wang and S. Dong, Nano Res., 2010, 3, 794.

25 H. Wang, K. Xie, Y. You, Q. Hou, K. Zhang, N. Li, W. Yu, K. P. Loh, C. Shen and B. Wei, Adv. Energy Mater., 2019, 9, 1901806.

26 A. Chen, X. Yu, Y. Zhou, S. Miao, Y. Li, S. Kuld, J. Sehested, J. Liu, T. Aoki and S. Hong, Nat. Catal., 2019, 2, 334.

27 G. Chen, C. Xu, X. Huang, J. Ye, L. Gu, G. Li, Z. Tang, B. Wu, H. Yang and Z. Zhao, Nat. Mater., 2016, 15, 564.

28 S. Chen, L. Li, W. Hu, X. Huang, Q. Li, Y. Xu, Y. Zuo and G. Li, ACS Appl. Mater. Interfaces, 2015, 7, 22999.

29 Y. Lin, L. Yang, Y. Zhang, H. Jiang, Z. Xiao, C. Wu, G. Zhang, J. Jiang and L. Song, Adv. Energy Mater., 2018, 8, 1703623.
30 C. Ye, Y. Jiao, D. Chao, T. Ling, J. Shan, B. Zhang, Q. Gu, K. Davey, H. Wang and S. Z. Qiao, Adv. Mater., 2020, 32, e1907557.

31 B. Delley, J. Chem. Phys., 2000, 113, 7756.

32 J. S. Yeon, S. Yun, J. M. Park and H. S. Park, ACS Nano, 2019, 13, 5163.

33 Z. Zhang, W. Li, M. F. Yuen, T.-W. Ng, Y. Tang, C.-S. Lee, X. Chen and W. Zhang, Nano Energy, 2015, 18, 196.

34 Z. L. Xu, S. Lin, N. Onofrio, L. Zhou, F. Shi, W. Lu, K. Kang, Q. Zhang and S. P. Lau, Nat. Commun., 2018, 9, 4164.

35 L. Zhang, X. Chen, F. Wan, Z. Niu, Y. Wang, Q. Zhang and J. Chen, ACS Nano, 2018, 12, 9578.

36 Y. Lin, L. Yang, Y. Zhang, H. Jiang, Z. Xiao, C. Wu, G. Zhang, J. Jiang and L. Song, Adv. Energy Mater., 2018, 8, 1703623.

37 W. Wang, Z. Cao, G. A. Elia, Y. Wu, W. Wahyudi, E. AbouHamad, A.-H. Emwas, L. Cavallo, L.-J. Li and J. Ming, ACS Energy Lett., 2018, 3, 2899.

38 D. Lv, J. Zheng, Q. Li, X. Xie, S. Ferrara, Z. Nie, L. B. Mehdi, N. D. Browning, J.-G. Zhang, G. L. Graff, J. Liu and J. Xiao, Adv. Energy Mater., 2015, 5, 1402290.

39 Z. G. Chen, J. Zou, D. W. Wang, L. C. Yin, G. Liu, Q. Liu, C. H. Sun, X. Yao, F. Li and X. L. Yuan, Adv. Funct. Mater., 2009, 19, 484.

40 B. Kang, S. Lim, W. H. Lee, S. B. Jo and K. Cho, Adv. Mater., 2013, 25, 5856.

41 J. Zhang, G. Yang, S. Wang, Y. Liu, Z. Zhao, Z. Wu, S. Zhang, X. Chen, C. Feng and G. Yu, J. Appl. Phys., 2014, 116, 163905. 